\title{
Instability of hairy black holes in spontaneously broken Einstein-Yang-Mills-Higgs systems
}

\author{
E. Winstanley ${ }^{a}$, N.E. Mavromatos ${ }^{b, 1}$ \\ ${ }^{a}$ Dept. of Physics (Theoretical Physics), University of Oxford, I Keble Road, Oxford OXI 3NP, UK \\ ${ }^{b}$ Laboratoire de Physique Thèorique ENSLAPP (URA 14-36 du CNRS, associée à l' E.N.S de Lyon, et au LAPP (IN2P3-CNRS) \\ d'Annecy-le-Vieux), Chemin de Bellevue, BP 110, F-74941 Annecy-le-Vieux Cedex, France
}

Received 19 March 1995

Editor: L. Alvarez-Gaumé

\begin{abstract}
The stability of a new class of hairy black-hole solutions in the coupled system of Einstein-Yang-Mills-Higgs is examined, generalising a method suggested by Brodbeck and Straumann and collaborators, and Volkov and Gal'tsov. The method maps the algebraic system of linearised radial perturbations of the various field modes around the black-hole solution into a coupled system of radial equations of Schrödinger type. No detailed knowledge of the black-hole solution is required, except from the fact that the boundary conditions at the physical space-time boundaries (horizons) must be such so as to guarantee the finiteness of the various expressions involved. In this way, it is demonstrated that the above Schrödinger equations have bound states, which implies the instability of the associated black-hole solution.
\end{abstract}

Coupling gravity to non-linear systems, such as the non-Abelian Yang-Mills theory, or the non-linear $\sigma$ models, etc., has led to interesting (classical) solutions with particle-like [1] or black-hole interpretation [2]. The interest in the latter type of solutions arises mainly from the fact that new types of classical hair have been shown to exist, contrary to the nohair conjecture characterising purely gravitational or Abelian black holes [3]. This is so, because the nohair theorems do not involve the issue of stability of the solutions in their proof, and therefore in this respect the above classical solutions may be considered as explicit counter examples to these theorems.

\footnotetext{
${ }^{1}$ On leave from P.P.A.R.C. Advanced Fellowship, Dept. of Physics (Theoretical Physics), University of Oxford, 1 Keble Road, Oxford OX1 3NP, UK.
}

In view of this, it is natural to enquire into the stability of the above solutions, which would establish their physical significance. It has been shown that most of these systems, especially the ones admitting particlelike interpretation, are unstable under perturbations of the various field modes [4]. For the black-hole solutions, a corresponding general proof was lacking so far, mainly due to the peculiar behaviour of the stability equations on the horizons. In some cases, however, like the Einstein-Yang-Mills-Higgs (EYMH) systems with a Higgs triplet, the Einstein-Skyrme (non-linear $\sigma$-model) system, and the Einstein-YangMills-dilaton theory (inspired from strings), linear stability of the hairy solutions is established (see, for instance, Ref. [5]), although non-linear stability remains an unsettled issue.

An interesting class of classical hairy black holes 
has been found recently in connection with the SU(2)-Einstein-Higgs system, with a Higgs doublet as in the standard model [6]. These black-hole solutions resemble the sphaleron solutions in SU(2) gauge theory and one would expect them to be unstable for topological reasons.

Recently, an instability proof of sphaleron solutions for arbitrary gauge groups in the EYM system has been given $[7,8]$. The method consists of studying linearised radial perturbations around an equilibrium solution, whose detailed knowledge is not necessary to establish stability. The stability is examined by mapping the system of algebraic equations for the perturbations into a coupled system of differential equations of Schrödinger type $[7,8]$. As in the particle case of Ref. [1], the instability of the solution is established once a bound state in the respective Schrödinger equations is found. The latter shows up as an imaginary frequency mode in the spectrum, leading to an exponentially growing mode. There is an elegant physical interpretation behind this analysis, which is similar to the Cooper pair instability of super-conductivity. The gravitational attraction balances the non-Abelian gauge field repulsion in the classical solution [1], but the existence of bound states implies imaginary parts in the quantum ground state which lead to instabilities of the solution, in much the same way as the classical ground state in super-conductivity is not the absolute minimum of the free energy.

However, this method cannot be applied directly to the black-hole case, due to divergences occurring in some of the expressions involved. This is a result of the singular behaviour of the metric function at the physical space-time boundaries (horizon) of the black hole.

It is the purpose of this note to generalise the method of Ref. [7] to incorporate the black-hole solution of the EYMH system of Ref. [6]. By constructing appropriate trial linear radial perturbations, following Refs. $[8,9]$, we show the existence of bound states in the spectrum of the coupled Schrödinger equations, and thus the instability of the black hole. Detailed knowledge of the black-hole solution is not aclually required, apart from the fact that the existence of an horizon leads to modifications of the trial perturbations as compared to those of Refs. [7,8], in order to avoid divergences in the respective expressions [9].

We start by sketching the basic steps $[7,9]$ that will lead to a study of the stability of a classical solution $\phi_{s}(x, t)$ with finite energy in a (generic) classical field theory. One considers small perturbations $\delta \phi(x, t)$ around $\phi_{s}(x, t)$, and specifies [7] the time dependence as

$\delta \phi(x, t)=\exp (-i \Omega t) \Psi(x)$.

The linearised system (with respect to such perturbations), obtained from the equations of motion, can be cast into a Schrödinger eigenvalue problem

$\mathcal{H} \Psi=\Omega^{2} A \Psi$

where the operators $\mathcal{H}, A$ are assumed independent of the "frequency" $\Omega$. As we shall show later on, this is indeed the case of our black-hole solution of the EYMH system. In that case it will also be shown that $\mathcal{H}$ is a self-adjoint operator with respect to a properly defined inner (scalar) product in the space of functions $\{\Psi\}[7]$, and the $A$ matrix is positive definite, $\langle\Psi|A| \Psi\rangle>0$. A criterion for instability is the existence of an imaginary frequency mode in (2)

$\Omega^{2}<0$.

This is usually difficult to solve analytically in realistic models, and usually numerical calculations are required [4]. A less informative method which admits analytic treatment has been proposed recently in Refs. [7,9], and we shall follow this for the purposes of the present work. The method consists of a variational approach which makes use of the following functional defined through (2):

$\Omega^{2}(\Psi)=\frac{\langle\Psi|\mathcal{H}| \Psi\rangle}{\langle\Psi|A| \Psi\rangle}$

with $\Psi$ a trial function. The lowest eigenvalue is known to provide a lower bound for this functional. Thus, the criterion of instability, which is equivalent to $(3)$, in this approach reads

$$
\begin{gathered}
\Omega^{2}(\Psi)<0, \\
\langle\Psi|A| \Psi\rangle<\infty .
\end{gathered}
$$

The first of the above conditions implies that the operator $\mathcal{H}$ is not positive definite, and therefore negative eigenvalues do exist. The second condition, on the finiteness of the expectation value of the operator $A$, is 
required to ensure that $\Psi$ lies in the Hilbert space containing the domain of $\mathcal{H}$. In certain cases, cspecially in the black-hole case, there are divergences due to singular behaviour of modes at, say, the horizons, which could spoil these conditions (5). The advantage of the above variational method lies in the fact that it is an easier task to choose appropriate trial functions $\Psi$ that satisfy (5) than solving the original eigenvalue problem (2). In what follows we shall apply this second method to the black-hole solution of Ref. [6].

We start by reviewing the basic formulas for a study of stability issues of spherically symmetric black-hole solutions of the EYMH system [6]. The space-time metric takes the form [6]

$$
\begin{aligned}
\mathrm{d} s^{2}= & -N(t, r) S^{2}(t, r) \mathrm{d} t^{2}+N^{-1} \mathrm{~d} r^{2} \\
& +r^{2}\left(\mathrm{~d} \theta^{2}+\sin ^{2} \theta \mathrm{d} \phi^{2}\right)
\end{aligned}
$$

and we assume the following ansatz for the nonAbelian gauge potential $[6,7]$ :

$$
\begin{aligned}
A= & a_{0} \tau_{r} \mathrm{~d} t+a_{1} \tau_{r} \mathrm{~d} r+(\omega-1)\left[\tau_{\phi} \mathrm{d} \theta-\tau_{\theta} \sin \theta \mathrm{d} \phi\right] \\
& +\tilde{\omega}\left[\tau_{\theta} \mathrm{d} \theta+\tau_{\phi} \sin \theta \mathrm{d} \phi\right],
\end{aligned}
$$

where $\omega, \tilde{\omega}$ and $a_{i}, i=0,1$ are functions of $t, r$. The $\tau_{i}$ are appropriately normalised spherical generators of the SU(2) group in the notation of Ref. [7].

The Higgs doublet assumes the form

$\Phi \equiv \frac{1}{\sqrt{2}}\left(\begin{array}{c}\psi_{2}+i \psi_{1} \\ \phi-i \psi_{3}\end{array}\right) ; \quad \psi=\psi r$

with the Higgs potential

$V(\Phi)=\frac{\lambda}{4}\left(\Phi^{\dagger} \Phi-v^{2}\right)^{2}$,

where $v$ denotes the v.e.v. of $\Phi$ in the non-trivial vacuum.

The quantities $\omega, \phi$ satisfy the static field equations

$$
\begin{aligned}
& N \omega^{\prime \prime}+\frac{(N S)^{\prime}}{S} \omega^{\prime} \\
& =\frac{1}{r^{2}}\left(\omega^{2}-1\right) \omega+\frac{\phi^{2}}{4}(\omega-1), \\
& N \phi^{\prime \prime}+\frac{(N S)^{\prime}}{S} \phi^{\prime}+\frac{2 N}{r} \phi^{\prime} \\
& =\frac{1}{2 r^{2}} \phi(\omega-1)^{2}+\lambda \phi\left(\phi^{2}-v^{2}\right),
\end{aligned}
$$

where the prime denotes differentiation with respect to $r$. For later usc, we also mention that a dot will denote differentiation with respect to $t$.

If we choose a gauge in which $\delta a_{0}=0$, the linearised perturbation equations decouple into two sectors [7]. The first consists of the gravitational modes $\delta N, \delta S, \delta \omega$ and $\delta \phi$ and the second of the matter perturbations $\delta a_{1}, \delta \tilde{\omega}$ and $\delta \psi$. In our analysis it will be sufficient to concentrate on the matter perturbations, setting the gravitational perturbations $\delta N$ and $\delta S$ to zero, because an instability will show up in this sector of the theory. The equations for the linearised matter perturbations take the form [7]

$\mathcal{H} \Psi+A \ddot{\Psi}=0$

with

$\Psi=\left(\begin{array}{c}\delta a_{1} \\ \delta \tilde{\omega} \\ \delta \psi\end{array}\right)$,

and

$A=\left(\begin{array}{ccc}N r^{2} & 0 & 0 \\ 0 & 2 & 0 \\ 0 & 0 & r^{2}\end{array}\right)$

and the components of $\mathcal{H}$ are

$$
\begin{aligned}
\mathcal{H}_{a_{1} a_{1}}= & 2(N S)^{2}\left(\omega^{2}+\frac{r^{2}}{8} \phi^{2}\right), \\
\mathcal{H}_{\tilde{\omega} \tilde{\omega}}= & 2 p_{*}^{2}+2 N S^{2}\left(\frac{\omega^{2}-1}{r^{2}}+\frac{\phi^{2}}{4}\right), \\
\mathcal{H}_{\psi \psi}= & 2 p_{*} \frac{r^{2}}{2} p_{*}+2 N S^{2} \\
& \times\left(\frac{(\omega+1)^{2}}{4}+\frac{r^{2}}{2} \lambda\left(\phi^{2}-v^{2}\right)\right), \\
\mathcal{H}_{a_{1} \tilde{\omega}}= & 2 i N S\left[\left(p_{*} \omega\right)-\omega p_{*}\right], \\
\mathcal{H}_{\tilde{\omega} a_{1}}= & 2 i\left[p_{*} N S \omega+N S\left(p_{*} \omega\right)\right], \\
\mathcal{H}_{a_{1} \psi}= & \left.\frac{i r^{2}}{2} N S \Gamma\left(p_{*} \phi\right)-\phi p_{*}\right], \\
\mathcal{H}_{\psi a_{1}}= & i p_{*} \frac{r^{2}}{2} N S \phi+i \frac{r^{2}}{2} N S\left(p_{*} \phi\right), \\
\mathcal{H}_{\tilde{\omega} \psi}= & \mathcal{H}_{\psi \tilde{\omega}}=-\phi N S^{2},
\end{aligned}
$$

where the operator $p_{*}$ is

$p_{*} \equiv-i N S \frac{\mathrm{d}}{\mathrm{d} r}$. 
Upon specifying the time dependence (1)

$\Psi(r, t)=\Psi(r) \mathrm{e}^{i \Omega t} ; \quad \Psi(r)=\left(\begin{array}{c}\delta a_{1}(r) \\ \delta \tilde{\omega}(r) \\ \delta \psi(r)\end{array}\right)$

one arrives easily to an eigenvalue problem of the form (2), which can then be extended to the variational approach (5).

To this end, we choose as trial perturbations the following expressions (cf. [7]):

$$
\begin{aligned}
\delta a_{1} & =\omega^{\prime} Z, \\
\delta \tilde{\omega} & =\left(\omega^{2}-1\right) Z, \\
\delta \psi & =\frac{1}{2} \phi(\omega-1) Z,
\end{aligned}
$$

where $Z$ is a function of $r$ to be determined.

One may define the inner product

$$
\langle\Psi \mid \Phi\rangle \equiv \int_{r_{l}}^{\infty} \bar{\Psi} \Phi \frac{1}{N S} \mathrm{~d} r,
$$

where $r_{h}$ is the position of the horizon of the black hole. The operator $\mathcal{H}$ is then symmetric with respect to this scalar product. Following Ref. [7], consider the expectation value

$$
\begin{aligned}
\langle\Psi|A| \Psi\rangle= & \int_{r_{h}}^{\infty} \mathrm{d} r \frac{1}{N S} Z^{2}\left[N r^{2}\left(\omega^{\prime}\right)^{2}+2\left(\omega^{2}-1\right)^{2}\right. \\
& \left.+\frac{r^{2}}{4} \phi^{2}(\omega-1)^{2}\right]
\end{aligned}
$$

which is clearly positive definite for real $Z$. Its finiteness will be examined later, and depends on the choice of the function $Z$.

Next, we proceed to the evaluation of the expectation value of the Hamiltonian $\mathcal{H}(14)$; after a tedious calculation one obtains

$$
\begin{aligned}
\langle\Psi|\mathcal{H}| \Psi\rangle= & \int_{r_{h}}^{\infty} \mathrm{d} r S Z^{2}\left\{-2 N\left(\omega^{\prime}\right)^{2}+2 P^{2} N\left(\omega^{2}-1\right)^{2}\right. \\
& +\frac{1}{4} P^{2} N r^{2} \phi^{2}(\omega-1)^{2}-\frac{2}{r^{2}}\left(\omega^{2}-1\right)^{2} \\
& \left.-\frac{1}{2} \phi^{2}(\omega-1)^{2}\right\}+ \text { boundary terms }
\end{aligned}
$$

where $P \equiv \mathrm{d} Z / Z \mathrm{~d} r$. The boundary terms will be shown to vanish so we omit them in the expression (19). The final result is

$$
\begin{aligned}
\langle\Psi|\mathcal{H}| \Psi\rangle= & \int_{r_{h}}^{\infty} \mathrm{d} r S\left\{-2 N\left(\omega^{\prime}\right)^{2}-\frac{2}{r^{2}}\left(\omega^{2}-1\right)^{2}\right. \\
& \left.-\frac{1}{2} \phi^{2}(\omega-1)^{2}\right\} \\
& +\int_{r_{h}}^{\infty} \mathrm{d} r\left\{\frac{2}{r^{2}}\left(\omega^{2}-1\right)^{2}\right. \\
& \left.+\phi^{2}(\omega-1)^{2}+2 N\left(\omega^{\prime}\right)^{2}\right\} S\left(1-Z^{2}\right) \\
& +\int_{r_{h}}^{\infty} \mathrm{d} r S N\left(\frac{\mathrm{d} Z}{\mathrm{~d} r}\right)^{2} \\
& \times\left[2\left(\omega^{2}-1\right)^{2}+\frac{1}{4} r^{2} \phi^{2}(\omega-1)^{2}\right] .
\end{aligned}
$$

The first of these terms is manifestly negative. To examine the remaining two, we introduce the "tortoise" co-ordinate $r^{*}$ defined by [9]

$$
\frac{\mathrm{d} r^{*}}{\mathrm{~d} r}=\frac{1}{N S}
$$

and define a sequence of functions $Z_{k}\left(r^{*}\right)$ by [9]

$$
Z_{k}\left(r^{*}\right)=Z\left(\frac{r^{*}}{k}\right) ; \quad k=1,2, \ldots,
$$

where

$$
\begin{aligned}
Z\left(r^{*}\right) & =Z\left(-r^{*}\right), \\
Z\left(r^{*}\right) & =1 \text { for } r^{*} \in[0, a], \\
-D \leqslant \frac{\mathrm{d} Z}{\mathrm{~d} r^{*}}<0 & \text { for } r^{*} \in[a, a+1], \\
Z\left(r^{*}\right)=0 & \text { for } r^{*}>a+1,
\end{aligned}
$$

where $a, D$ are arbitrary positive constants. Then, for each value of $k$ the vacuum expectation values of $\mathcal{H}$ and $A$ are finite, $\langle\Psi|\mathcal{H}| \Psi\rangle\langle\infty$, and $\langle\Psi|A| \Psi\rangle\langle\infty$, with $Z=Z_{k}$, and all boundary terms vanish. This justifies a posteriori their being dropped in Eq. (19). The integrands in the second and third terms of Eq. (20) 
are uniformly convergent and tend to zero as $k \rightarrow \infty$. Hence, choosing $k$ sufficiently large the dominant contribution in (20) comes from the first term which is negative.

This confirms the existence of bound states in the Schrödinger equations (11), (2), and thereby the instability (5) of the associated black-hole solution of Ref. [6] in the coupled EYMH system.

The above analysis reveals the existence of at least one negative odd-parity eigenmode in the spectrum of the EYMH black hole, which implies its instability. The exact number of such negative modes is an interesting question and we plan to investigate it in the near future. Recently, a method for determining the number of the sphaleron-like unstable modes has been applied by Volkov et al. [10] to the gravitating sphaleron case, and one might be able to extend it to the present EYMH black hole. According to the analysis of Ref. [10], for EYM black holes, there are $n$ unstable sphaleron-like modes under radial perturbations, where $n$ is the number of nodes of the equilibrium solution [1]. This number does not depend on the details of the equilibrium solution, such as the horizon geometry, size, etc. This is due to the topological nature of the instabilities. In this respect, we mention that an interesting connection could be made with the global analysis of Ref. [11], where catastrophe theory was invoked to provide a way of evaluating the number of unstable modes of certain (non-sphaleron) blackhole solutions. From the global analysis of Ref. [11] there are other non-sphaleronic types of non-Abelian black holes, whose "high entropy" phase is stable. In our analysis, this would imply an extension of the variational approach to incorporate finite temperature effects for the matter perturbations in non-sphaleron black holes ${ }^{2}$. The finite temperature would be a result of the existence of the horizon entropy associated with the black hole in a semi-classical analysis. It might well be that the number of unstable modes of these (non-sphaleron) black holes is somehow affected by the temperature, in the sense that above a "critical" temperature (corresponding to a certain horizon size)

\footnotetext{
${ }^{2}$ For sphaleron-like black holes, the topological nature of the instability might complicate the connection with catastrophe theory if the number of unstable modes is independent of the details of the equilibrium solution, as appears to be the case for the EYM system [10].
}

the bound states of the Schrödinger equation (2) disappear, or their number is reduced. This would correspond to the high-entropy "stable" black holes of Ref. [11], in the sense of the catastrophe theory. At present, such issues remain open. We hope to come back to these in the near future.

We thank K. Tamvakis and P. Kanti for discussions. One of us (E.W.) would like to thank CERN, Theory Division, for the hospitality during the initial stages of this work. She also thanks E.P.S.R.C. (U.K.) for a research studentship. The work of N.E.M. is supported by a EC Research Fellowship, Proposal Nr. ERB4001GT922259.

\section{References}

[1] R. Bartnik and J. McKinnon, Phys. Rev. Lett. 6 I (1988) 141.

[2] P. Bizon, Phys. Rev. Lett. 64 (1990) 2644

[3] C. Misner, K. Thorne and J.A. Wheeler, Gravitation (Freeman, San Francisco, 1973);

J. Bekenstein, Phys. Rev. D 5 (1972) 1239;

S. Adler and R. Pearson, Phys. Rev. D 18 (1978) 2798.

[4] N. Straumann and Z.H. Zhou, Phys. Lett. B 237 (1990) 353; B 243 (1991) 53; Nucl. Phys. B 369 (1991) 180.

[5] M. Heusler, N. Straumann and Z.H. Zhou, Helv. Phys. Acta 66 (1993) 614;

K-Y. Lee, V.P. Nair and E. Weinberg, Phys. Rev. Lett. 68 (1992) 1100;

M.E. Ortiz, Phys. Rev. 45 (1992) R2586;

P. Breitenholder, P. Forgács and D. Maison, Nucl. Phys. B 383 ( 1992) 357;

E.E. Donets and D. Gal'tsov, Phys. Lett. B 302 (1993) 411; P. Bizon, Act. Phys. Pol. B 24 (1993) 1209.

[6] B.R. Greene, S.D. Mathur and C.M. O'Neill, Phys. Rev. D 47 (1993) 2242.

[7] P. Boschung, O. Brodbeck, F. Moser, N. Straumann and M. Volkov, Phys. Rev. 50 (1994) 3842

[8] O. Brodbeck and N. Straumann, Zürich ETH preprint ZUTH 38/94 (1994), bulletin no: gr-qc/9411058.

[9] M. Volkov and D. Gal'tsov, Phys. Lett. B 341 (1995) 279.

[10] M.S. Volkov, O. Brodbeck, G. Lavrelashvili and N. Straumann, Zürich ETH preprint ZU-TH 3/95 (1995), bulletin no: hep-th/9502045.

[11] K. Maeda, T. Tachizava, T. Torii and T. Maki, Phys. Rev Lett. 72 (1994) 450 . 